\title{
Article \\ Characteristics and Lenvatinib Treatment Response of Unresectable Hepatocellular Carcinoma with Iso-High Intensity in the Hepatobiliary Phase of EOB-MRI
}

\author{
Akinori Kubo ${ }^{1,+}$, Goki Suda ${ }^{1, *,+}$, Megumi Kimura ${ }^{1}$, Osamu Maehara ${ }^{2}$, Yoshimasa Tokuchi ${ }^{1}$, \\ Takashi Kitagataya ${ }^{1}$, Masatsugu Ohara ${ }^{1}$, Ren Yamada ${ }^{1}$, Taku Shigesawa ${ }^{1}$, Kazuharu Suzuki ${ }^{1}$, Naoki Kawagishi ${ }^{1}$, \\ Masato Nakai ${ }^{1}$, Takuya Sho ${ }^{1}$, Mitsuteru Natsuizaka ${ }^{1}$, Kenichi Morikawa ${ }^{1}$, Koji Ogawa ${ }^{1}$, Shunsuke Ohnishi ${ }^{2}$ (D) \\ and Naoya Sakamoto ${ }^{1}$
}

Citation: Kubo, A.; Suda, G.; Kimura M.; Maehara, O.; Tokuchi, Y.; Kitagataya, T.; Ohara, M.; Yamada, R.; Shigesawa, T.; Suzuki, K.; et al. Characteristics and Lenvatinib Treatment Response of Unresectable Hepatocellular Carcinoma with Iso-High Intensity in the Hepatobiliary Phase of EOB-MRI. Cancers 2021, 13, 3633. https:// doi.org/10.3390/cancers13143633

Academic Editor: Alessandro Vitale

Received: 5 July 2021

Accepted: 16 July 2021

Published: 20 July 2021

Publisher's Note: MDPI stays neutral with regard to jurisdictional claims in published maps and institutional affiliations.

Copyright: (C) 2021 by the authors Licensee MDPI, Basel, Switzerland. This article is an open access article distributed under the terms and conditions of the Creative Commons Attribution (CC BY) license (https:/ / creativecommons.org/licenses/by/ $4.0 /$ )
1 Departments of Gastroenterology and Hepatology, Graduate School of Medicine, Hokkaido University, Sapporo 060-8589, Japan; kubo.akinori.q5@elms.hokudai.ac.jp (A.K.); myamakam@med.hokudai.ac.jp (M.K.); tokuchi.yoshimasa.e2@elms.hokudai.ac.jp (Y.T.); t.kitagataya@pop.med.hokudai.ac.jp (T.K.); masamasama_zu@yahoo.co.jp (M.O.); renyama5@pop.med.hokudai.ac.jp (R.Y.); Tshigesawa@pop.med.hokudai.ac.jp (T.S.); kazuharu-s@hospital.hakodate.hokkaido.jp (K.S.); naopaleg@yahoo.co.jp (N.K.); mnakai@pop.med.hokudai.ac.jp (M.N.); shotaku@pop.med.hokudai.ac.jp (T.S.); mitsuteru@natsuizakaclinic.com (M.N.); kenichi.morikawa@med.hokudai.ac.jp (K.M.); k-ogawa@med.hokudai.ac.jp (K.O.); sakamoto@med.hokudai.ac.jp (N.S.)

2 Laboratory of Molecular and Cellular Medicine, Faculty of Pharmaceutical Sciences, Hokkaido University, Sapporo 060-8589, Japan; maeosa17@frontier.hokudai.ac.jp (O.M.); sonishi@pop.med.hokudai.ac.jp (S.O.)

* Correspondence: gsudgast@pop.med.hokudai.ac.jp; Tel.: +81-11-716-1161

$\dagger \quad$ The authors equally contributed to this study.

Simple Summary: HCC with alterations in CTNNB1 (which encodes $\beta$-catenin) is resistant to immune checkpoint inhibitors and is associated with HCC with iso-high intensity in the hepatobiliary phase of EOB-MRI in resectable HCC. However, the prevalence, characteristics, mutation profile, and treatment response in unresectable HCC with iso-high intensity in the hepatobiliary phase of EOB-MRI are not well clarified. In this study, we showed that the prevalence was $13 \%$, and the response to lenvatinib does not differ between HCC with and without iso-high intensity in the hepatobiliary phase of EOB-MRI. We analyzed CTNNB-1 mutations using cell-free DNA, providing support for their association with iso-high intensity in the hepatobiliary phase of EOB-MRI.

Abstract: In hepatocellular carcinoma (HCC), CTNNB-1 mutations, which cause resistance to immune checkpoint inhibitors, are associated with HCC with iso-high intensity in the hepatobiliary phase of gadoxetic acid-enhanced magnetic resonance imaging (EOB-MRI) in resectable HCC; however, analyses on unresectable HCC are lacking. This study analyzed the prevalence, characteristics, response to lenvatinib, and CTNNB-1 mutation frequency in unresectable HCC with iso-high intensity in the hepatobiliary phase of EOB-MRI. In 52 patients with unresectable HCC treated with lenvatinib, the prevalence of iso-high intensity in the hepatobiliary phase of EOB-MRI was $13 \%$. All patients had multiple HCCs, and 3 patients had multiple HCCs with iso-high intensity in the hepatobiliary phase of EOB-MRI. Lenvatinib response to progression-free survival and overall survival were similar between patients with or without iso-high intensity in the hepatobiliary phase of EOBMRI. Seven patients (three and four patients who had unresectable HCC with or without iso-high intensity in the hepatobiliary phase of EOB-MRI, respectively) underwent genetic analyses. Among these, two $(67 \%, 2 / 3)$ who had HCC with iso-high intensity in the hepatobiliary phase of EOB-MRI carried a CTNNB-1 mutation, while all four patients who had HCC without iso-high intensity in the hepatobiliary phase of EOB-MRI did not carry the CTNNB-1 mutation. This study's findings have clinical implications for the detection and treatment of HCC with iso-high intensity in the hepatobiliary phase of EOB-MRI.

Keywords: CTNNB-1; HCC with iso-high intensity in the hepatobiliary phase of EOB-MRI; Lenvatinib 


\section{Introduction}

Recent advances have dramatically changed the hepatocellular carcinoma (HCC) treatment landscape. The development of immune checkpoint inhibitors (ICIs), which target negative anti-cancer immune response regulators, has drastically improved anticancer therapy. Various clinical trials and real-world data have revealed the efficacy of ICIs, conferring a durable response, which is not observed in conventional anti-cancer therapy, in various malignancies [1-5]. A clinical trial on HCC has revealed that bevacizumab plus atezolizumab has been associated with better overall survival (OS) in patients with unresectable tumors than sorafenib, which was the standard therapy for patients with unresectable HCC [6].

However, some patients did not experience sufficient treatment response. Thus, predictive factors for response to ICIs have been an important clinical issue and identified in various malignancies, including PD-L1 expression, immune cell infiltration, mutation burden, $\mathrm{T}$ cell receptor repertoires, and the gut microbiome [7]. Recently, Harding et al. reported that in patients with advanced HCC carrying WNT/ $\beta$-catenin mutations, ICI treatment results in a lower disease control rate and shorter median OS than those for HCC without $W N T / \beta$-catenin mutations [8]. Thus, the presence of $W N T / \beta$-catenin mutations in advanced HCC might be an effective predictive factor for resistance to ICI therapy. However, analyses of genetic alterations in advanced HCC are difficult because biopsy of advanced HCC sometimes causes tumor dissemination and bleeding. Thus, alternative methods for the detection of $\mathrm{WNT} / \beta$-catenin mutations in advanced $\mathrm{HCC}$ are required. HCC imaging using gadoxetic acid-enhanced magnetic resonance imaging (EOB-MRI) could be a candidate approach. In the hepatobiliary phase of EOB-MRI, HCC typically shows low intensity, while HCC with the WNT/ $\beta$-catenin mutation shows iso-high intensity because WNT / $\beta$-catenin mutations result in higher organic anion transporter 1B3 (OATP1B3) expression than in surrounding non-HCC hepatocytes [9-11]. In resectable HCC, the reported frequency of cases with iso-high intensity in the hepatobiliary phase of EOB-MRI is $12 \%(22 / 180)$ to $20 \%(5 / 25)$ [12-15]. However, the prevalence of unresectable HCC with iso-high intensity in the hepatobiliary phase of EOB-MRI, requiring systemic therapy, has not been clarified.

Analyses of cell-free DNA (cfDNA) are promising for detecting genetic alterations in HCC $[16,17]$ and may provide insight into the frequent mutations in unresectable HCC with high intensity in the hepatobiliary phase of EOB-MRI.

Lenvatinib is a recently developed multi-kinase inhibitor that targets VEGFR1-3, FGFR1-4, RET c-Kit, and PDGFR- $\alpha$ mediated signaling $[18,19]$. The REFLECT trial, which is a phase 3 clinical trial regarding lenvatinib for patients with unresectable HCC, revealed that lenvatinib has similar OS compared with sorafenib, while lenvatinib has superior progression-free survival than sorafenib [20]. Real-world data validated the efficacy and safety of lenvatinib for patients with unresectable HCC [21,22]. Thus, in patients with unresectable HCC, who might have a poor response to ICIs, lenvatinib could be alternative first-line therapy. However, the efficacy and safety of lenvatinib in patients with unresectable HCC with iso-high intensity in the hepatobiliary phase of EOB-MRI have not been clarified.

Therefore, in this study, we analyzed (a) the prevalence of unresectable HCC with iso-high intensity in the hepatobiliary phase of EOB-MRI in patients with unresectable HCC who were treated with lenvatinib, (b) the response to lenvatinib, and (c) the relationship between the presence of unresectable HCC with iso-high intensity in the hepatobiliary phase of EOB-MRI and $\beta$-Catenin (CTNNB-1) mutations determined by a cfDNA analysis.

\section{Materials and Methods}

\subsection{Patients and Study Design}

Patients with unresectable HCC for whom lenvatinib treatment was initiated at Hokkaido University Hospital between April 2018 and April 2021 were screened in this retrospective study. We included patients if they had an EOB-MRI examination before 
lenvatinib treatment and were evaluated after treatment using dynamic computed tomography (CT) or EOB-MRI at baseline and every $2-3$ months. Patients were excluded if they had decompensated liver cirrhosis, had insufficient clinical data or no EOB-MRI examination, had a treatment duration of less than 1 week, or were not evaluated for treatment responses by dynamic CT or MRI.

Attending physicians routinely assessed patients using laboratory tests and physical examinations every 2 weeks and using enhanced CT or EOB-MRI every 2-3 months after treatment initiation.

\subsection{Treatment Protocol}

Patients were administrated lenvatinib according to the package insert as follows: once-daily doses of 8 and $12 \mathrm{mg}$ for patients weighing $<60$ and $\geq 60 \mathrm{~kg}$, respectively.

\subsection{Evaluation of HCC with Iso-High Intensity in the Hepatobiliary Phase of EOB-MRI}

Two MRI systems, Achieva $1.5 \mathrm{~T}$ dStream (Philips N. V., Tokyo, Japan) and TRILLIUMOVAL 3.0 T (HITACHI, Tokyo, Japan), were utilized for the evaluation of HCC according to previously described methods [10] by two hepatologists. The signal intensities (SIs) of the tumor and surrounding non-tumor liver tissues were measured by defining regions of interest (ROIs). The enhancement in HCC relative to liver parenchyma was calculated according to a previously described method [15] as follows. Relative intensity ratio $($ RIR $)=$ SI (nodule) $/$ SI (parenchyma). Relative enhancement ratio $($ RER) $=$ RIR (in hepatobiliary-phase images)/RIR (precontract RIR). Tumor enhancement was defined as iso-high when RER $\geq 1.0$.

\subsection{Evaluation of the Response to Lenvatinib}

Treatment responses were evaluated every 2-3 months using dynamic CT based on mRECIST criteria [23].

\subsection{CTNNB1 Mutation Detection in Cell-Free DNA}

Eleven of the 52 patients included had cfDNA at baseline and/or within 1 month after treatment initiation. Two of these patients had HCC with iso-high intensity in the hepatobiliary phase of EOB-MRI. Moreover, four patients without HCC with iso-high intensity in the hepatobiliary phase of EOB-MRI who initiated lenvatinib in June 2019 and agreed to cfDNA collection were selected for an analysis of CTNNB1 alterations. In addition, CTNNB1 alterations in cfDNA were analyzed in another patient who had unresectable HCC with iso-high intensity in the hepatobiliary phase of EOB-MRI and had cfDNA.

For the extraction of cfDNA, $4 \mathrm{~mL}$ of plasma was collected, and cfDNA was extracted using the AVENIO cfDNA Isolation Kit (Roche Diagnostics K. K., Tokyo, Japan) according to the manufacturer's protocol at Tsukuba i-Laboratory LLP (Tsukuba, Ibaraki, Japan) and stored at $-80^{\circ} \mathrm{C}$. The concentration of cfDNA was determined using the Qubit dsDNA HS Assay Kit (Thermo Fisher Scientific, Waltham, MA, USA), and fragment sizes were examined using the Agilent 2200 TapeStation System with High Sensitivity D5000 ScreenTape Assay (Agilent, Santa Clara, CA, USA).

We conducted library preparation and sequencing according to the manufacturer's (Thermo Fisher Scientific) methods. We utilized an Oncomine Colon cfDNA for PCR amplification of target regions, and library preparation for sequencing reads was mapped using Torrent Suite 5.6.0 (Thermo Fisher Scientific). Variant calling was performed using Oncomine Colon Liquid Biopsy-w2.1-DNA-Single Sample workflow in Ion Reporter version 5.6 (Thermo Fisher Scientific). Modified Oncomine_Colon_cfDNA.03062017, a BED file, was used for variant calling at hotspot sites. Annotations of called variants were based on dbSNP151, CCDS (NCBI, Release 15), RefSeq (UCSC Genome Browser, Nov 2018), Gencode (UCSC Genome Browser, ver. 19), and 1000 Genomes (phase3 release v5). 


\subsection{Statistical Analysis}

Categorical variables were analyzed using the chi-square and Fisher's exact tests. Continuous variables were analyzed using the Mann-Whitney U-test. In this study, we set statistical significance at $p<0.05$.

Progression-free survival (PFS) and overall survival (OS) were defined as follows; the time from the initiation of lenvatinib to the day of disease progression or last follow-up and death, respectively. PFS and OS curves were compared using the log-rank test. The association of etiology and overall survival was analyzed by Cox regression analysis. Statistical analyses were performed using IBM SPSS Statistics 22.0 (IBM Corp., Armonk, NY, USA).

\section{Results}

\subsection{Enrolled Patients and Baseline Characteristics}

Between April 2018 and April 2021, a total of 61 patients with unresectable HCC were treated with lenvatinib at Hokkaido University Hospital. Among these patients, nine were excluded owing to a lack of EOB-MRI examination and/or insufficient treatment duration. Finally, 52 patients with unresectable HCC were included in the study (Figure 1).

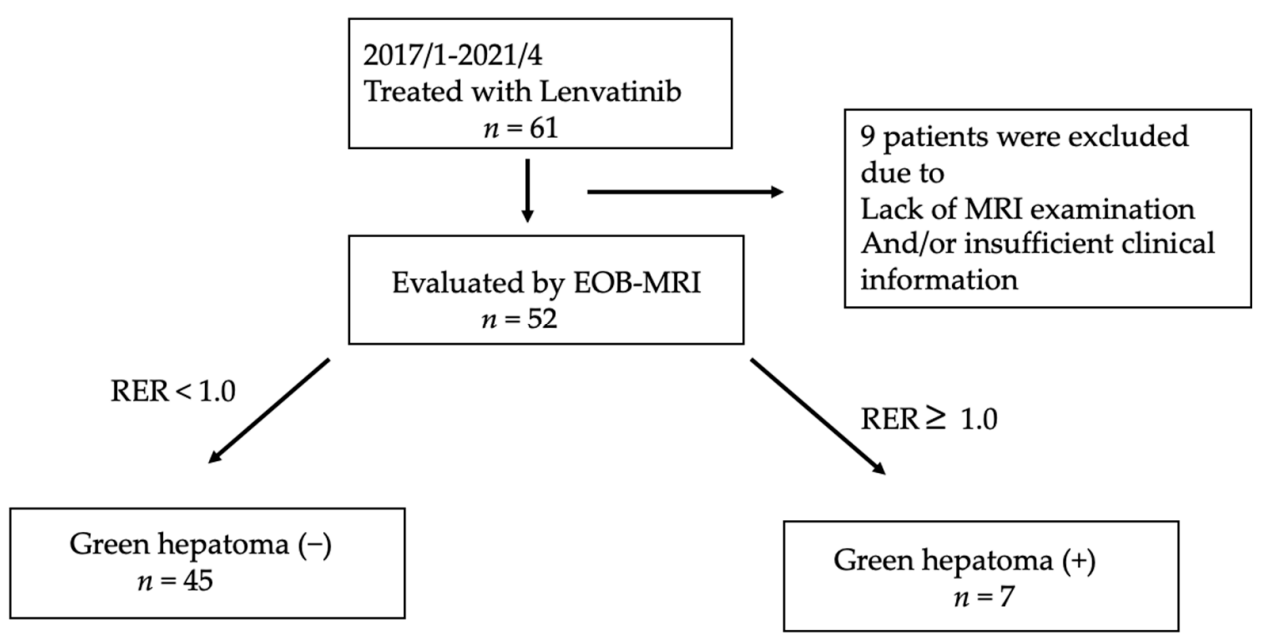

Figure 1. Study Flow.

Table 1 shows the baseline characteristics of patients. The median age of patients with HCC with iso-high intensity in the hepatobiliary phase of EOB-MRI was 70 years and that of patients without was 71 years. A total of $88 \%$ of patients $(46 / 52)$ were males. Forty-one and 11 patients had Child-Pugh grades A and B, respectively. Twenty-seven and 25 patients had BCLC stages B and C, respectively. The median serum AFP and PIVKA-II levels were $56.7 \mathrm{IU} / \mathrm{mL}$ (range, 1.6-449,909 IU/mL) and $404.0 \mathrm{mAU} / \mathrm{mL}$ (range, $13-416,670 \mathrm{mAU} / \mathrm{mL}$ ), respectively. Regarding disease etiology, 14, 8, and 30 patients had $\mathrm{HBV}$ infection, $\mathrm{HCV}$ infection, and non-B non-C liver disease. 
Table 1. Characteristics of patients with unresectable HCC with or without iso-high intensity in the hepatobiliary phase of EOB-MRI.

\begin{tabular}{|c|c|c|c|c|}
\hline $\begin{array}{c}\text { Clinical } \\
\text { Characteristics }\end{array}$ & $\begin{array}{c}\text { Patients with HCC } \\
\text { Treated by Lenvatinib } \\
(n=52)\end{array}$ & $\begin{array}{c}\text { Green Hepatoma }(n= \\
7)\end{array}$ & $\begin{array}{l}\text { Green Hepatoma }(-) \\
\qquad(n=45)\end{array}$ & $p$-Value \\
\hline \multicolumn{5}{|l|}{ Baseline characteristics } \\
\hline Age (yr) & $70(45-83)$ & $70(55-79)$ & $71(45-83)$ & 0.9357 \\
\hline Sex (Male/Female) & $46 / 6$ & $7 / 0$ & $39 / 6$ & 0.3043 \\
\hline \multicolumn{5}{|l|}{ Etiology, No. (\%) } \\
\hline $\mathrm{HBV}$ & $14(26.9 \%)$ & $0(0 \%)$ & $14(31.1 \%)$ & 0.0702 \\
\hline $\mathrm{HCV}$ & $8(15.3 \%)$ & $1(14.3 \%)$ & $7(15.5 \%)$ & 0.9310 \\
\hline NBNC & $30(57.6 \%)$ & $6(85.7 \%)$ & $24(53.3 \%)$ & 0.0864 \\
\hline $\begin{array}{c}\text { Vascular invasion, No. } \\
(\%)\end{array}$ & $16(30.7 \%)$ & $0(0.0 \%)$ & $16(35.5 \%)$ & 0.1006 \\
\hline $\begin{array}{c}\text { Extrahepatic extension, } \\
\text { No. }(\%)\end{array}$ & $16(30.7 \%)$ & $0(0.0 \%)$ & $16(35.5 \%)$ & 0.0580 \\
\hline \multicolumn{5}{|l|}{ BCLC stage, No. (\%) } \\
\hline B & $27(51.9 \%)$ & $5(71.4 \%)$ & $22(48.8 \%)$ & 0.2669 \\
\hline $\mathrm{C}$ & $25(48.0 \%)$ & $2(28.6 . \%)$ & $23(51.1 \%)$ & \\
\hline \multicolumn{5}{|l|}{$\begin{array}{c}\text { Child-Pugh class, No. } \\
(\%)\end{array}$} \\
\hline $\mathrm{A}$ & $41(78.8 \%)$ & $4(57.1 \%)$ & $37(82.2 \%)$ & 0.1307 \\
\hline B & $11(21.1 \%)$ & $3(42.8 \%)$ & $8(17.7 \%)$ & \\
\hline \multicolumn{5}{|l|}{ Biochemical analysis } \\
\hline Albumin, $\mathrm{g} / \mathrm{dL}$ & $3.7(2.8-4.6)$ & $3.2(2.9-4.6)$ & $3.8(2.8-4.6)$ & 0.4354 \\
\hline Total bilirubin, $\mathrm{mg} / \mathrm{dL}$ & $0.7(0.3-3.1)$ & $1.2(0.9-3.1)$ & $0.7(0.3-1.9)$ & 0.0197 \\
\hline Prothrombin time, $\%$ & $88.3(40.7-117.7)$ & $83.9(46.6-107.6)$ & $88.4(40.7-117.7)$ & 0.1637 \\
\hline Platelet, $\times 10^{4} / \mu \mathrm{L}$ & $16.1(4.4-51.7)$ & $13.4(4.4-51.7)$ & $17.3(6.5-51.7)$ & 0.4448 \\
\hline $\begin{array}{c}\text { Alpha-fetoprotein, } \\
\text { ng/mL }\end{array}$ & $78.3(2.0-449,909)$ & $2.2(2.2-852.5)$ & $78.3(2.0-449,909)$ & 0.4759 \\
\hline AFP, L3\% & $19.2(0-99.5)$ & $41.1(0.5-80.4)$ & $19.2(0-99.5)$ & 0.4273 \\
\hline PIVKA-II, mAU/mL & $345(13-93,644)$ & $266(26-29,756)$ & $381(13-93,644)$ & 0.3961 \\
\hline RER & $0.785(0.446-1.446)$ & $1.110(1.018-1.446)$ & $0.7526(0.446-0.969)$ & $<0.001$ \\
\hline
\end{tabular}

yr, years; HBV, hepatitis B virus; HCV, hepatitis C virus; NBNC, non-B non-C; BCLC, Barcelona Clinic Liver Cancer; AFP, alpha-fetoprotein; PIVKA-II, protein induced by vitamin K absence or antagonist-II; RER, relative enhancement ratio of nodule; Green hepatoma, HCC with iso-high intensity in the hepatobiliary phase of EOB-MRI.

3.2. Prevalence and Characteristics of HCC with Iso-High Intensity in the Hepatobiliary Phase of EOB-MRI in Patients with Unresectable HCC Who Were Treated with Lenvatinib

Overall, among 52 patients, 7 (13\%) had HCC with high intensity in the hepatobiliary phase of EOB-MRI. Typical MR images are shown in Figure 2. As summarized in Table 1 , the median age, sex, BCLC stage, and tumor markers were similar in patients with unresectable HCC with or without iso-high intensity in the hepatobiliary phase of EOBMRI. Table 2 presents the characteristics of patients with unresectable HCC with iso-high intensity in the hepatobiliary phase of EOB-MRI. All patients had multiple HCCs and had both HCCs with or without iso-high intensity in the hepatobiliary phase of EOB-MRI simultaneously. Three patients $(42.9 \%)$ had multiple HCCs with iso-high intensity in the hepatobiliary phase of EOB-MRI. 


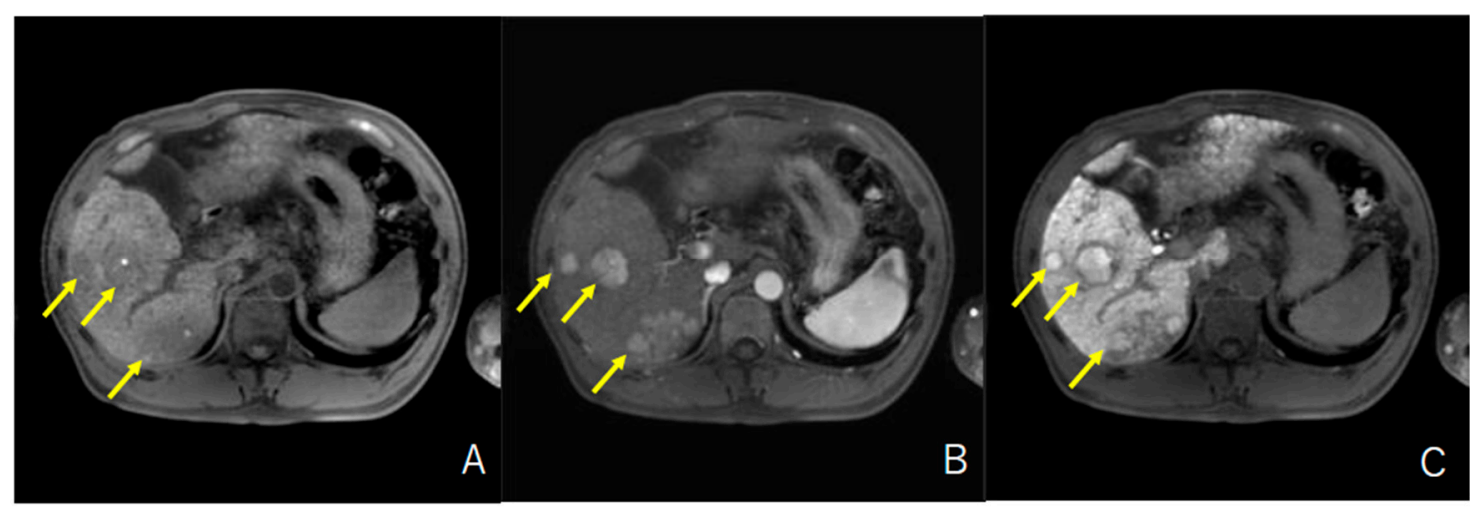

Figure 2. Typical MR images of unresectable HCC with iso-high intensity in the hepatobiliary phase. (A) Plain phase, (B) Arterial phase, (C) Hepatobiliary phase.

Table 2. Characteristics of patients with HCC with iso-high intensity in the hepatobiliary phase of EOB-MRI.

\begin{tabular}{|c|c|c|c|c|c|c|c|}
\hline Pt No. & Age (years) & Sex & $\begin{array}{c}\text { HCCs in Liver } \\
(n)\end{array}$ & $\begin{array}{c}\text { Green } \\
\text { Hepatoma }(n)\end{array}$ & $\begin{array}{c}\text { Size of Green } \\
\text { Hepatoma } \\
(\mathrm{mm})\end{array}$ & $\begin{array}{c}\text { RER } \\
\text { (Median) }\end{array}$ & Etiology \\
\hline 1 & 55 & M & 6 & 3 & $12.6,33.3,32.5$ & 1.155 & $\mathrm{HCV}$ \\
\hline 2 & 79 & M & 5 & 2 & $11.1,19.1$ & 1.174 & NBNC \\
\hline 3 & 70 & M & 3 & 2 & $16.6,39.1$ & 1.191 & NBNC \\
\hline 4 & 73 & M & 3 & 1 & 10 & 1.073 & NBNC \\
\hline 5 & 67 & M & 4 & 1 & 18.4 & 1.085 & NBNC \\
\hline 6 & 83 & M & 5 & 1 & 19.8 & 1.105 & NBNC \\
\hline 7 & 57 & M & 4 & 1 & 23.6 & 1.004 & NBNC \\
\hline
\end{tabular}

RER, relative enhancement ratio of nodule; $y r$, years; $M$, Male; HCV, hepatitis C virus; NBNC, non-B non-C.

\subsection{Treatment Response, Overall Survival, and Progression-Free Survival}

Subsequently, we analyzed the response to lenvatinib, PFS, and OS in patients with unresectable HCC. As shown in Table 3, treatment responses were similar between patients with or without HCC with iso-high intensity in the hepatobiliary phase of EOB-MRI; the objective response rates (ORR) were $42.8 \%$ (3/7) and $48.8 \%(22 / 45)$, and the disease control rates (DCR) were $100 \%(7 / 7)$ and $86.6 \%(39 / 45)$ in patients with or without HCC with iso-high intensity in the hepatobiliary phase of EOB-MRI, respectively.

Table 3. Comparison of the response to lenvatinib between patients with or without unresectable HCC with or without iso-high intensity in the hepatobiliary phase of EOB-MRI imaging.

\begin{tabular}{cccc}
\hline Treatment Response & $\begin{array}{c}\text { Green Hepatoma } \\
\boldsymbol{n}=\mathbf{7}\end{array}$ & $\begin{array}{c}\text { Green Hepatoma (-) } \\
\boldsymbol{n}=\mathbf{4 5}\end{array}$ & $\boldsymbol{p}$-Value \\
\hline $\mathrm{CR} / \mathrm{PR} / \mathrm{SD} / \mathrm{PD}$ & $0 / 3 / 4 / 0$ & $4 / 19 / 16 / 6$ & 0.5087 \\
$\mathrm{ORR}$ & $3(42.8 \%)$ & $22(48.8 \%)$ & 0.5901 \\
$\mathrm{DCR}$ & $7(100 \%)$ & $39(86.6 \%)$ & 0.3416 \\
\hline
\end{tabular}

CR, complete response; $\mathrm{PR}$, partial response; $\mathrm{SD}$, stable disease; $\mathrm{PD}$, progressive disease; ORR, objective response rate; DCR, disease control rate.

Comparison of OS and PFS in patients with HCC with or without iso-high intensity in the hepatobiliary phase of EOB-MRI treated with lenvatinib are shown in Figure 3. PFS rates were not significantly different between patients with or without HCC with iso-high intensity in the hepatobiliary phase of EOB-MRI (median PFS; 7.0 and 8.3 months, respectively; $\mathrm{HR}=1.041074,95 \%$ CI: 0.863166-1.171921, $p=0.8456$ ). Similarly, OS rates were not significantly different between patients with or without HCC with iso-high intensity in the hepatobiliary phase of EOB-MRI (median OS; 17.5 and 19.5 months, respectively; HR = 1.1449, 95\% CI: 0.892235-1.090671, $p=0.8315)$. In addition, as shown in the Supplementary Table S1, cox regression analysis revealed that the presence of HCC with iso-high intensity 
in the hepatobiliary phase of EOB-MRI did not affect OS (HR 0.95, 95\% CI 0.153-4.849, $p=0.9613)$.

A

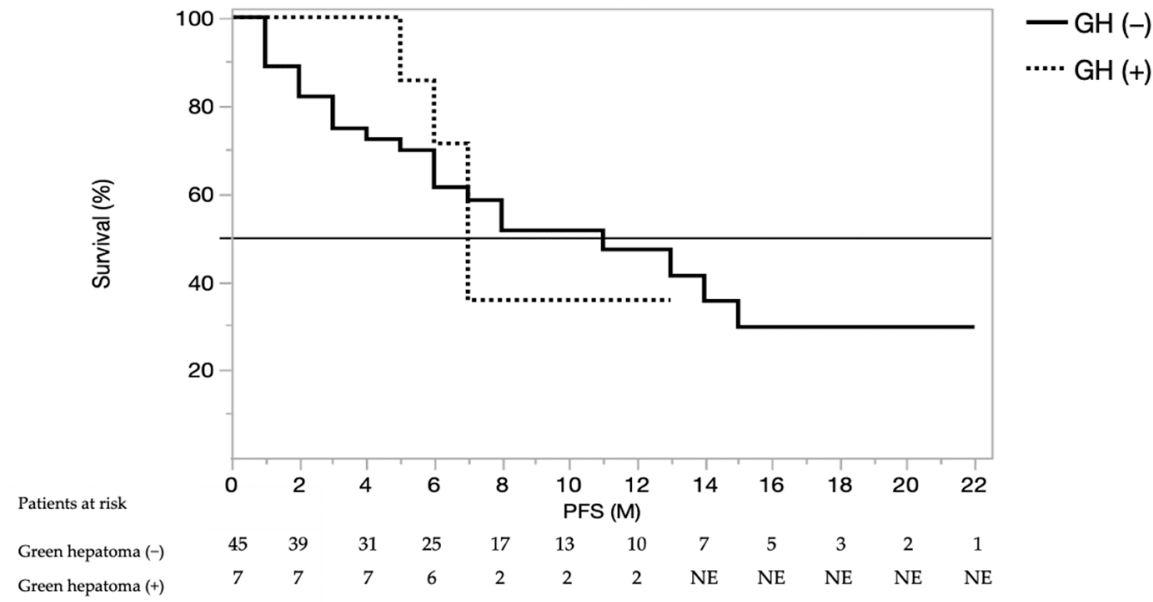

B

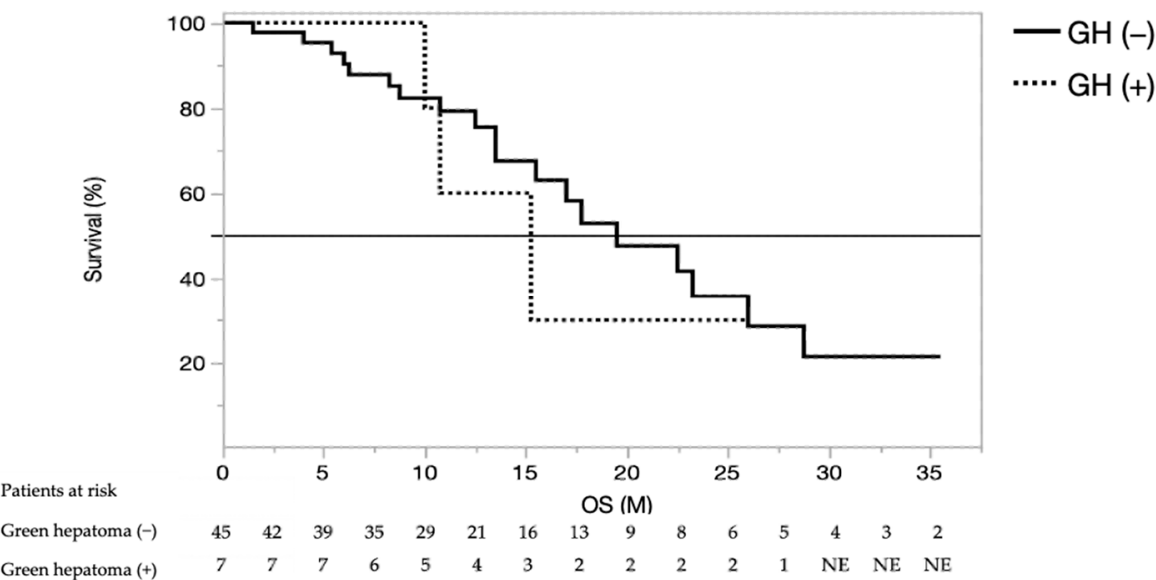

Figure 3. Kaplan-Meier estimates of (A) overall survival (OS) and (B) progression-free survival (PFS) in patients with unresectable HCC with or without iso-high intensity in the hepatobiliary phase of EOB-MRI. M, month; NE, not evaluated; GH, Green hepatoma.

3.4. Relationship between HCC with Iso-High Intensity in the Hepatobiliary Phase of EOB-MRI and WNT/B-Catenin Mutations Diagnosed by Cell-Free DNA

Finally, we analyzed the CTNNB1 mutation profile in cfDNA in one patient with unresectable HCC with iso-high intensity in the hepatobiliary phase of EOB-MRI and three patients without HCC with iso-high intensity in the hepatobiliary phase of EOB-MRI who were treated with lenvatinib.

As shown in Table 4, of three the patient with HCC with iso-high intensity in the hepatobiliary phase of EOB-MRI, two $(66.7 \% 2 / 3)$ showed CTNNB-1 alterations (case 1; CTNNB-1 T41A, variant frequency 30/13,721; CTNNB-1 S45F, variant frequency 28/13,723, case 2; CTNNB-1 T41A, variant frequency 196/12,329) in cfDNA. 
Table 4. CTNNB-1 gene alterations in cell-free DNA in five patients with or without iso-high intensity in the hepatobiliary phase of EOB-MRI.

\begin{tabular}{ccccccccc}
\hline Case & $\begin{array}{c}\text { Green } \\
\text { Hepatoma }\end{array}$ & $\begin{array}{c}\text { Age } \\
\text { (Years) }\end{array}$ & Sex & Etiology & $\begin{array}{c}\text { Child- } \\
\text { Pugh }\end{array}$ & BCLC & CTNNB-1 Mutation \\
\hline 1 & + & 70 & M & NBNC & 6 & B & positive & T41AS45F \\
2 & + & 72 & M & NBNC & 6 & B & positive & T41A \\
3 & + & 83 & M & NBNC & 6 & B & negative \\
4 & - & 70 & M & NBNC & 5 & B & negative \\
5 & - & 62 & M & NBNC & 6 & B & negative \\
6 & - & 70 & M & NBNC & 7 & B & negative \\
7 & - & 70 & F & NBNC & 5 & B & ne \\
\hline
\end{tabular}

BCLC, Barcelona clinic liver cancer; NBNC, non-B-non-C.

In the four patients with HCC without iso-high intensity in the hepatobiliary phase of EOB-MRI, CTNNB-1 genetic alterations were not detected in cfDNA.

\section{Discussion}

In this study, we revealed that the prevalence of HCC with high intensity in the hepatobiliary phase of EOB-MRI was $13.4 \%$ (7/52) among patients with unresectable HCC. All 7 patients had multiple HCCs and had both HCCs with or without iso-high intensity in the hepatobiliary phase of EOB-MRI simultaneously. Only 3 patients had multiple HCCs with iso-high intensity in the hepatobiliary phase of EOB-MRI. The response to lenvatinib, PFS, and OS was similar between patients with or without iso-high intensity in the hepatobiliary phase of EOB-MRI. Furthermore, our results supported the relationship between iso-high intensity in the hepatobiliary phase of EOB-MRI and CTNNB-1 mutations detected in cfDNA.

The wnt/ $\beta$-catenin pathway is known to be involved in the development of HCC. Hoshida et al. reported that HCC with WNT/ $\beta$-catenin mutations is associated with good survival, pathologically well-differentiated tumors, smaller tumor sizes, and the S3 subclass [24]. Similarly, Zucman-Rossi et al. reported that HCC with WNT/ $\beta$-catenin mutations is pathologically well-differentiated, lacks inflammatory infiltrates, and is classified as G5 and G6 subclass [25]. CTNNB1 gene alterations in HCC are associated with less lymphocytic infiltration [25] and decreased levels of CCL5, which recruits antigen-specific CD8+ T cells, resulting in poor immunogenicity and resistance to ICI therapy [11,26]. Thus, HCC with CTNNB1 alterations is called "cold tumors" or "immune desert tumors" [27]. Importantly, a recent report has revealed that HCC with mutant CTNNB-1 has a poor response to ICI treatment, resulting in a poor prognosis [8].

However, because tumor biopsy is an invasive procedure in patients with unresectable HCC, it is difficult to obtain samples for genetic analyses. Thus, EOB-MRI is a potential alternative. In HCC, WNT/ $\beta$-catenin mutations induce the upregulation of OATP1B3, which transports Gd-EOBDTPA [10], resulting in high intensity in the hepatobiliary phase of EOB-MRI [10]. Thus, HCC with iso-high intensity in the hepatobiliary phase of EOB-MRI indicates the presence of $\mathrm{WNT} / \beta$-catenin mutations.

A previous study revealed that the prevalence of HCC with iso-high intensity in the hepatobiliary phase of EOB-MRI is $12 \%(22 / 180)$ to $20 \%(5 / 25)$ in typical resectable HCC [12-15]. To the best of our knowledge, this is the first report of the prevalence in patients with unresectable HCC who are adapted to systemic chemotherapy. Of 52 patients with unresectable HCC treated with lenvatinib, a total of $13 \%$ of patients had HCC with iso-high intensity in the hepatobiliary phase of EOB-MRI. This prevalence was similar to that reported in resectable HCC. However, all patients had multiple HCCs with and without iso-high intensity in the hepatobiliary phase of EOB-MRI. Only three patients had multiple HCCs with iso-high intensity in the hepatobiliary phase of EOB-MRI. This result might reflect the nature of multicentric carcinogenesis in HCC. This should be considered when evaluating the results of this study. 
Comparison between patients with or without HCCs with iso-high intensity in the hepatobiliary phase of EOB-MRI revealed that in patients with HCCs with iso-high intensity in the hepatobiliary phase of EOB-MRI, the prevalence of HCC etiology of HBV had a low tendency ( $0 \%$ vs. $26.9 \% p=0.0702$ ), while the prevalence of HCC etiology of non-B non-C had a high tendency $(85.7 \%$ vs. $53.3 \%, p=0.0864)$. This result might be consistent with the previous report that the nonproliferation class of HCC had a high prevalence of WNT/b-catenin activation and etiology of HCV and alcohol, not that of HBV [28].

In this study, the response to lenvatinib (as evaluated by the DCR and ORR) was similar for patients with iso-high intensity in the hepatobiliary phase of EOB-MRI and without. In addition, OS and PFS were similar between patients with or without HCC with iso-high intensity in the hepatobiliary phase of EOB-MRI. These results are consistent with those of a previous report showing that sorafenib is similarly effective for patients with or without advanced HCC harboring a WNT/ $\beta$-catenin mutation and that PFS and median OS after ICI treatment are lower in HCC with mutant $W N T / \beta$-catenin than with wild-type WNT/ $\beta$-catenin [8]. Thus, in patients with unresectable HCC, EOB-MRI might be useful for selecting appropriate therapies. However, further studies are required, especially studies of the responses of unresectable HCC with iso-high intensity in the hepatobiliary phase of EOB-MRI to atezolizumab plus bevacizumab.

Finally, our analysis supported the relationship between unresectable HCC with high intensity in the hepatobiliary phase of EOB-MRI and CTNNB-1 mutations determined by cfDNA analysis. The analysis of cfDNA is an effective method for the detection of genetic alterations in HCC $[16,29,30]$, and it is less impacted by intratumoral heterogeneity than are analyses of single tumor tissue samples [31]. In three patients with unresectable HCC with high intensity in the hepatobiliary phase of EOB-MRI, CTNNB1 alterations in cfDNA were observed in two patients $(66.7 \%, 2 / 3)$, while all four patients without HCC with iso-high intensity in the hepatobiliary phase of EOB-MRI lacked mutations. Thus, CTNNB1 alterations may be associated with HCC with iso-high intensity in the hepatobiliary phase of EOB-MRI in patients with resectable and unresectable HCC. In one patient with HCC with high intensity in the hepatobiliary phase of EOB-MRI, the CTNNB1 alteration in cfDNA was not detected. The reason why CTNNB1 alteration in cfDAA was not detected has not been clarified. Sensitivity of NGS analysis, quality of sample, or existence of another gene alteration of WNT/b-catenin pathway such as AXIN 1 may have been the reasons. Additionally, the number of patients was limited; thus, further studies of a larger number of patients are required.

Moreover, the prevalence of CTNNB1 alterations in advanced HCC is reported to be $35.7 \%$ [8]. Thus, patients without HCC, iso-high intensity in the hepatobiliary phase of EOB-MRI might have CTNNB1 alterations. Additional analyses of cfDNA for the detection of CTNNB1 alteration are needed. However, in advanced HCC, multicentric carcinogenesis is usually observed, resulting in different genetic alterations within the same patient $[32,33]$. Thus, it is not clear whether genetic alterations in cfDNA represent all HCCs, and the results of genetic analyses should be interpreted with caution.

There are several limitations in this study. It was a retrospective single-center study and included a relatively small sample size, especially for patients who had HCC with iso-high intensity in the hepatobiliary phase of EOB-MRI. Moreover, the number of patients with an analysis for the genetic alteration of CTNNB-1 in cfDNA was quite limited. Thus, those limitations should be considered when interpreting the results. However, this study is the first to estimate the prevalence of unresectable HCC with iso-high intensity in the hepatobiliary phase of EOB-MRI and its association with CTNNB1 mutations and the response to lenvatinib. Therefore, future prospective multicenter studies, including large sample sizes, should be required to validate the results of this study.

\section{Conclusions}

We revealed that the prevalence of HCC with iso-high intensity in the hepatobiliary phase of EOB-MRI in patients with unresectable HCC is $13.4 \%(7 / 52)$. Further- 
more, we found that unresectable HCC with iso-high intensity in the hepatobiliary phase of EOB-MRI is related to CTNNB-1 mutations in cfDNA, consistent with previous results for resectable HCC. The response to lenvatinib, PFS, and OS were similar in patients with or without iso-high intensity in the hepatobiliary phase of EOB-MRI.

Supplementary Materials: The following are available online at https://www.mdpi.com/article/10 .3390 / cancers13143633/s1: Table S1; Cox regression analysis of association between clinical factors and overall survival.

Author Contributions: A.K. and G.S. contributed equally to this work. A.K. and G.S. designed this study, performed the statistical analyses, and wrote the manuscript. M.K., O.M., Y.T., T.K., M.O., R.Y., T.S. (Taku Shigesawa), K.S., N.K., M.N. (Masato Nakai), T.S. (Takuya Sho), M.N. (Mitsuteru Natsuizaka), K.M., K.O. and S.O. collected the data. S.O. and N.S. provided hepatological advice and edited the manuscript. N.S. revised the manuscript for important intellectual content. All authors have read and agreed to the published version of the manuscript.

Funding: This study was supported in part by grants from the Japan Agency for Medical Research and Development (AMED; grant number JP20fk0210072, JP20fk0210064, JP20fk0210056, JP20fk0310101, JP20fk0210047, JP20fk0210048: JP20fk0210058, JP20fk0210066, JP20fk0210067) and SPS KAKENHI (grant No. 19K08458).

Institutional Review Board Statement: The study protocol conformed to the ethical guidelines of the Declaration of Helsinki and was approved by the Hokkaido University Hospital ethics committee (Approval No. 017-0521).

Informed Consent Statement: All included patients provided informed consent to participate in this study.

Data Availability Statement: The data that support the findings of this study are available from the corresponding author upon reasonable request.

Acknowledgments: The authors want to thank all patients and their families, as well as the investigators and staff of the participating institution.

Conflicts of Interest: Naoya Sakamoto has received lecture fees from Bristol Myers Squibb and Pharmaceutical K.K., grants and endowments from MSD, K.K., and Chugai Pharmaceutical Co., Ltd., and a research grant from Gilead Sciences Inc. and AbbVie GK. Goki Suda received research grants from Merck \& Co., Gilead Sciences Inc., and Bristol Myers Squibb. The other authors have no conflicts of interest to disclose.

\section{References}

1. Roth, D.; Nelson, D.R.; Bruchfeld, A.; Liapakis, A.; Silva, M.; Monsour, H., Jr.; Martin, P.; Pol, S.; Londono, M.C.; Hassanein, T.; et al. Grazoprevir plus elbasvir in treatment-naive and treatment-experienced patients with hepatitis $C$ virus genotype 1 infection and stage 4-5 chronic kidney disease (the C-SURFER study): A combination phase 3 study. Lancet 2015, 386, 1537-1545. [CrossRef]

2. Mazza, C.; Escudier, B.; Albiges, L. Nivolumab in renal cell carcinoma: Latest evidence and clinical potential. Ther. Adv. Med. Oncol. 2017, 9, 171-181. [CrossRef]

3. Fehrenbacher, L.; Spira, A.; Ballinger, M.; Kowanetz, M.; Vansteenkiste, J.; Mazieres, J.; Park, K.; Smith, D.; Artal-Cortes, A.; Lewanski, C.; et al. Atezolizumab versus docetaxel for patients with previously treated non-small-cell lung cancer (POPLAR): A multicentre, open-label, phase 2 randomised controlled trial. Lancet 2016, 387, 1837-1846. [CrossRef]

4. Gandhi, L.; Rodriguez-Abreu, D.; Gadgeel, S.; Esteban, E.; Felip, E.; De Angelis, F.; Domine, M.; Clingan, P.; Hochmair, M.J.; Powell, S.F.; et al. Pembrolizumab plus Chemotherapy in Metastatic Non-Small-Cell Lung Cancer. N. Engl. J. Med. 2018, 378, 2078-2092. [CrossRef] [PubMed]

5. Motzer, R.J.; Tannir, N.M.; McDermott, D.F.; Aren Frontera, O.; Melichar, B.; Choueiri, T.K.; Plimack, E.R.; Barthelemy, P.; Porta, C.; George, S.; et al. Nivolumab plus Ipilimumab versus Sunitinib in Advanced Renal-Cell Carcinoma. N. Engl. J. Med. 2018, 378, 1277-1290. [CrossRef] [PubMed]

6. $\quad$ Finn, R.S.; Qin, S.; Ikeda, M.; Galle, P.R.; Ducreux, M.; Kim, T.Y.; Kudo, M.; Breder, V.; Merle, P.; Kaseb, A.O.; et al. Atezolizumab plus Bevacizumab in Unresectable Hepatocellular Carcinoma. N. Engl. J. Med. 2020, 382, 1894-1905. [CrossRef]

7. Nakamura, Y. Biomarkers for Immune Checkpoint Inhibitor-Mediated Tumor Response and Adverse Events. Front. Med. 2019, 6, 119. [CrossRef] [PubMed] 
8. Harding, J.J.; Nandakumar, S.; Armenia, J.; Khalil, D.N.; Albano, M.; Ly, M.; Shia, J.; Hechtman, J.F.; Kundra, R.; El Dika, I.; et al. Prospective Genotyping of Hepatocellular Carcinoma: Clinical Implications of Next-Generation Sequencing for Matching Patients to Targeted and Immune Therapies. Clin. Cancer Res. 2019, 25, 2116-2126. [CrossRef] [PubMed]

9. Kitao, A.; Matsui, O.; Yoneda, N.; Kozaka, K.; Kobayashi, S.; Sanada, J.; Koda, W.; Minami, T.; Inoue, D.; Yoshida, K.; et al. Hepatocellular Carcinoma with beta-Catenin Mutation: Imaging and Pathologic Characteristics. Radiology 2015, $275,708-717$. [CrossRef]

10. Ueno, A.; Masugi, Y.; Yamazaki, K.; Komuta, M.; Effendi, K.; Tanami, Y.; Tsujikawa, H.; Tanimoto, A.; Okuda, S.; Itano, O.; et al. OATP1B3 expression is strongly associated with Wnt/beta-catenin signalling and represents the transporter of gadoxetic acid in hepatocellular carcinoma. J. Hepatol. 2014, 61, 1080-1087. [CrossRef] [PubMed]

11. Kudo, M. Gd-EOB-DTPA-MRI Could Predict WNT/beta-Catenin Mutation and Resistance to Immune Checkpoint Inhibitor Therapy in Hepatocellular Carcinoma. Liver Cancer 2020, 9, 479-490. [CrossRef] [PubMed]

12. Narita, M.; Hatano, E.; Arizono, S.; Miyagawa-Hayashino, A.; Isoda, H.; Kitamura, K.; Taura, K.; Yasuchika, K.; Nitta, T.; Ikai, I.; et al. Expression of OATP1B3 determines uptake of Gd-EOB-DTPA in hepatocellular carcinoma. J. Gastroenterol. 2009, 44, 793-798. [CrossRef] [PubMed]

13. Kitao, A.; Zen, Y.; Matsui, O.; Gabata, T.; Kobayashi, S.; Koda, W.; Kozaka, K.; Yoneda, N.; Yamashita, T.; Kaneko, S.; et al. Hepatocellular carcinoma: Signal intensity at gadoxetic acid-enhanced MR Imaging-correlation with molecular transporters and histopathologic features. Radiology 2010, 256, 817-826. [CrossRef]

14. Kitao, A.; Matsui, O.; Yoneda, N.; Kozaka, K.; Kobayashi, S.; Koda, W.; Gabata, T.; Yamashita, T.; Kaneko, S.; Nakanuma, Y.; et al. Hypervascular hepatocellular carcinoma: Correlation between biologic features and signal intensity on gadoxetic acid-enhanced MR images. Radiology 2012, 265, 780-789. [CrossRef] [PubMed]

15. Tsuboyama, T.; Onishi, H.; Kim, T.; Akita, H.; Hori, M.; Tatsumi, M.; Nakamoto, A.; Nagano, H.; Matsuura, N.; Wakasa, K.; et al. Hepatocellular carcinoma: Hepatocyte-selective enhancement at gadoxetic acid-enhanced MR imaging-correlation with expression of sinusoidal and canalicular transporters and bile accumulation. Radiology 2010, 255, 824-833. [CrossRef]

16. Chen, V.L.; Xu, D.; Wicha, M.S.; Lok, A.S.; Parikh, N.D. Utility of Liquid Biopsy Analysis in Detection of Hepatocellular Carcinoma, Determination of Prognosis, and Disease Monitoring: A Systematic Review. Clin. Gastroenterol. Hepatol. 2020, 18, 2879-2902.e2879. [CrossRef]

17. Cai, Z.; Chen, G.; Zeng, Y.; Dong, X.; Li, Z.; Huang, Y.; Xin, F.; Qiu, L.; Xu, H.; Zhang, W.; et al. Comprehensive Liquid Profiling of Circulating Tumor DNA and Protein Biomarkers in Long-Term Follow-Up Patients with Hepatocellular Carcinoma. Clin. Cancer Res. 2019, 25, 5284-5294. [CrossRef] [PubMed]

18. Yamamoto, Y.; Matsui, J.; Matsushima, T.; Obaishi, H.; Miyazaki, K.; Nakamura, K.; Tohyama, O.; Semba, T.; Yamaguchi, A.; Hoshi, S.S.; et al. Lenvatinib, an angiogenesis inhibitor targeting VEGFR/FGFR, shows broad antitumor activity in human tumor xenograft models associated with microvessel density and pericyte coverage. Vasc. Cell 2014, 6, 18. [CrossRef]

19. Eso, Y.; Marusawa, H. Novel approaches for molecular targeted therapy against hepatocellular carcinoma. Hepatol. Res. 2018, 48, 597-607. [CrossRef]

20. Kudo, M.; Finn, R.S.; Qin, S.; Han, K.H.; Ikeda, K.; Piscaglia, F.; Baron, A.; Park, J.W.; Han, G.; Jassem, J.; et al. Lenvatinib versus sorafenib in first-line treatment of patients with unresectable hepatocellular carcinoma: A randomised phase 3 non-inferiority trial. Lancet 2018, 391, 1163-1173. [CrossRef]

21. Sho, T.; Suda, G.; Ogawa, K.; Shigesawa, T.; Suzuki, K.; Nakamura, A.; Ohara, M.; Umemura, M.; Kawagishi, N.; Natsuizaka, M.; et al. Lenvatinib in patients with unresectable hepatocellular carcinoma who do not meet the REFLECT trial eligibility criteria. Hepatol. Res. 2020, 50, 966-977. [CrossRef] [PubMed]

22. Sho, T.; Suda, G.; Ogawa, K.; Kimura, M.; Shimazaki, T.; Maehara, O.; Shigesawa, T.; Suzuki, K.; Nakamura, A.; Ohara, M.; et al. Early response and safety of lenvatinib for patients with advanced hepatocellular carcinoma in a real-world setting. JGH Open 2020, 4, 54-60. [CrossRef] [PubMed]

23. Lencioni, R.; Llovet, J.M. Modified RECIST (mRECIST) assessment for hepatocellular carcinoma. Semin. liver Dis. 2010, 30, 52-60. [CrossRef] [PubMed]

24. Hoshida, Y.; Nijman, S.M.; Kobayashi, M.; Chan, J.A.; Brunet, J.P.; Chiang, D.Y.; Villanueva, A.; Newell, P.; Ikeda, K.; Hashimoto, M.; et al. Integrative transcriptome analysis reveals common molecular subclasses of human hepatocellular carcinoma. Cancer Res. 2009, 69, 7385-7392. [CrossRef]

25. Calderaro, J.; Couchy, G.; Imbeaud, S.; Amaddeo, G.; Letouze, E.; Blanc, J.F.; Laurent, C.; Hajji, Y.; Azoulay, D.; Bioulac-Sage, P.; et al. Histological subtypes of hepatocellular carcinoma are related to gene mutations and molecular tumour classification. $J$. Hepatol. 2017, 67, 727-738. [CrossRef]

26. Llovet, J.M.; Montal, R.; Sia, D.; Finn, R.S. Molecular therapies and precision medicine for hepatocellular carcinoma. Nat. Rev. Clin. Oncol. 2018, 15, 599-616. [CrossRef]

27. Sia, D.; Jiao, Y.; Martinez-Quetglas, I.; Kuchuk, O.; Villacorta-Martin, C.; Castro de Moura, M.; Putra, J.; Camprecios, G.; Bassaganyas, L.; Akers, N.; et al. Identification of an Immune-specific Class of Hepatocellular Carcinoma, Based on Molecular Features. Gastroenterology 2017, 153, 812-826. [CrossRef]

28. Zucman-Rossi, J.; Villanueva, A.; Nault, J.C.; Llovet, J.M. Genetic Landscape and Biomarkers of Hepatocellular Carcinoma. Gastroenterology 2015, 149, 1226-1239.e1224. [CrossRef] 
29. Jiao, J.; Watt, G.P.; Stevenson, H.L.; Calderone, T.L.; Fisher-Hoch, S.P.; Ye, Y.; Wu, X.; Vierling, J.M.; Beretta, L. Telomerase reverse transcriptase mutations in plasma DNA in patients with hepatocellular carcinoma or cirrhosis: Prevalence and risk factors. Hepatol. Commun. 2018, 2, 718-731. [CrossRef]

30. Xu, R.H.; Wei, W.; Krawczyk, M.; Wang, W.; Luo, H.; Flagg, K.; Yi, S.; Shi, W.; Quan, Q.; Li, K.; et al. Circulating tumour DNA methylation markers for diagnosis and prognosis of hepatocellular carcinoma. Nat. Mater. 2017, 16, 1155-1161. [CrossRef] [PubMed]

31. Dhayat, S.A.; Yang, Z. Impact of circulating tumor DNA in hepatocellular and pancreatic carcinomas. J. Cancer Res. Clin. Oncol. 2020, 146, 1625-1645. [CrossRef] [PubMed]

32. Fujimoto, A.; Totoki, Y.; Abe, T.; Boroevich, K.A.; Hosoda, F.; Nguyen, H.H.; Aoki, M.; Hosono, N.; Kubo, M.; Miya, F.; et al. Whole-genome sequencing of liver cancers identifies etiological influences on mutation patterns and recurrent mutations in chromatin regulators. Nat. Genet. 2012, 44, 760-764. [CrossRef] [PubMed]

33. Nakagawa, H.; Shibata, T. Comprehensive genome sequencing of the liver cancer genome. Cancer Lett. 2013, 340, 234-240. [CrossRef] [PubMed] 